\title{
DETECTING DAMAGE IN STEEL WITH SCANNING SQUID MICROSCOPY
}

\author{
Tae-Kyu Lee ${ }^{1}$, David Clatterbuck ${ }^{1}$, J.W. Morris, Jr. ${ }^{1}$, T.J. Shaw ${ }^{2}$, R. McDermott ${ }^{2}$ and John Clarke $\underline{2}$ \\ ${ }^{1}$ Dept. of Materials Science and Engineering, ${ }^{2}$ Dept. of Physics, University of California, Berkeley, CA 94720 \\ and Materials Sciences Division, Lawrence Berkeley Laboratory, Berkeley, CA 94720
}

\begin{abstract}
A "Holy Grail" of NDE research is a non-destructive method for measuring fatigue damage prior to crack initiation. High-Tc scanning SQUID microscopy may be a useful tool. Because of the exceptional magnetic sensitivity of this technique, fatigue damage can be detected well before microcrack initiation, and in the absence of other obvious microstructure or property changes. Given the spatial resolution of the technique, undamaged material can be located and used to set internal standards.
\end{abstract}

\section{INTRODUCTION}

One of the most formidable and important problems in non-destructive testing is the development of techniques to detect high-cycle fatigue damage prior to crack nucleation. In a typical example of high-cycle fatigue a material experiences repeated stress or strain cycles until cracks nucleate and propagate to failure. Detectable crack nucleation may not occur until more than $90 \%$ of the fatigue life has been expended. The problem is particularly severe in operating devices that experience a spectrum of cyclic loads. Since fatigue cracks can propagate under loads that are small compared to those required to nucleate them, and since crack growth accelerates geometrically as it proceeds, the "window in time" for crack detection between nucleation and catastrophic failure may be very short. It is, hence, of great importance to find ways to detect fatigue damage prior to crack nucleation.

High cycle fatigue is ordinarily divisible into three mechanistically distinct stages. The first stage lasts for about the first $10 \%$ of the fatigue life, and is characterized by significant changes in the dislocation density and structure, as the microstructure accommodates to the cyclic stress or strain. The second stage extends through roughly the next $80 \%$ of the fatigue life. In this stage the dislocation substructure evolves slowly and becomes increasingly heterogeneous, leading to the formation of the "persistent slip bands" that will eventually nucleate cracks. The third stage begins with the formation of fatigue cracks at about $90 \%$ of the fatigue life and ends with final failure.

Because significant microstructural changes occur in the first and third stages of fatigue, researchers have had some success in developing NDE methods that probe them. Magnetic methods are particularly promising for ferromagnetic materials such as structural steels $[1,2]$. Parameters such as the saturation magnetization, coercivity, Barkhausen noise and magnetic hysteresis change significantly during the initial and final stages of fatigue. Tests with sensitive SQUID (superconducting quantum interference device) magnetometers have identified characteristic changes in the piezomagnetic hysteresis that may be particularly useful [3-6]. 
However, these techniques suffer from two important shortcomings. First, they do not readily measure the progress of fatigue in stage 2 [2], which is the longest and, usually, the most important stage. (A part in stage 1 has most of its life left, and a part in stage 3 is, ordinarily, so close to failure that one cannot chance waiting for it to appear.) Second, these techniques do not provide reliable absolute measures of fatigue damage [7]. They detect fatigue by measuring changes in properties rather than the properties themselves and are, hence, only useful in situations where the affected parts can be tested periodically over time.

We have recently been investigating the applicability of a new technique, scanning SQUID microscopy, to the non-destructive evaluation of ferromagnetic materials such as structural steels [8-11]. This technique makes it possible to map the superficial magnetic field of a specimen with high precision and spatial resolution, revealing changes in the microstructural state of a material on a very fine scale. In prior work we have shown that SQUID microscopy can be used to distinguish the microstructural constituents of steel [10] and to identify gradients in plastic deformation (at least when these have associated gradients in the dislocation density [11]). In the present work we extended this investigation to high-cycle fatigue.

We are particularly interested in the potential of SQUID microscopy to detect fatigue damage prior to crack nucleation. For this reason we chose to investigate fatigue in "9Ni" steel, a high-alloy dislocated lath martensitic steel with excellent toughness and ductility. Many carbon steels and high-strength steels are liable to fine-scale microcracking as fatigue damage accumulates. "9Ni" steel is expected to be virtually immune to this kind of damage, making it possible to focus on the detection of the irreversible local plastic deformation which is the essential feature of fatigue damage in stage 2 .
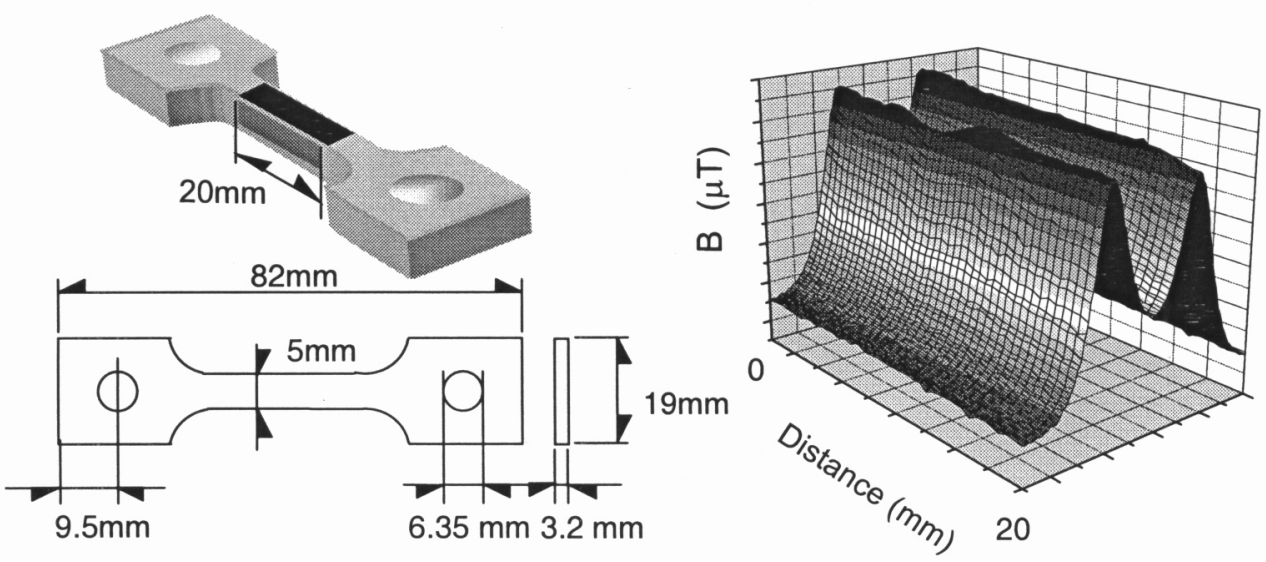

FIGURE 1. The geometry of the fatigue specimen and the SQUID magnetization map of a sample prior to fatigue testing.

\section{EXPERIMENTAL}

\section{$\underline{\text { Materials }}$}

The samples studied here were cut from a commercial plate of "9Ni" steel (Fe-9.3Ni$.05 \mathrm{C}-.28 \mathrm{Si}-.64 \mathrm{Mn}-.003 \mathrm{P}-.002 \mathrm{~S}-.027 \mathrm{Al}$, by weight) that was provided by the Pohang Iron and Steel Corporation (POSCO). The samples were machined into plate tensile specimens (Fig. 1). They were heat-treated with a modern version of the "QT" treatment [12]. They were annealed at $1300^{\circ} \mathrm{C}$ for $10 \mathrm{hr}$. to remove previous heat treatment, austenitized at $1170^{\circ} \mathrm{C}$ for 30 minutes, quenched to liquid nitrogen temperature to produce a fully martensitic microstructure, and finally given an intercritical temper at $590^{\circ} \mathrm{C}$ for $1 \mathrm{hr}$. Their surfaces were polished flat prior to testing. The samples were fatigued in a dynamic testing system (INSTRON 8500) under load control. The stress cycle was a tension-tension cycle 
from a stress near zero $(10 \mathrm{MPa})$ to $450 \mathrm{MPa}$ (about $70 \%$ of the yield strength) or $600 \mathrm{MPa}$ (almost $95 \%$ of yield).

\section{SQUID Microscope Apparatus and Measurements}

The SQUID microscope is shown in Fig. 2 and described in detail in refs. [8] and [10]. A high- $T_{c}$, thin film DC SQUID magnetometer is placed inside a vacuum enclosure and thermally coupled to a liquid nitrogen reservoir by a cold finger. It sits just below a sealed, $125 \mu \mathrm{m}$ thick sapphire window. A sample stage rests on a large brass table and is attached to a two-dimensional (2D) translation stage. It contains three height adjustment screws for alignment purposes. The sample is mounted on a mylar film attached to the sample stage while a weighted finger holds the sample against the vacuum window, maintaining a constant SQUID-to-sample separation. A computer raster scans the sample stage over the SQUID with stepper motors while recording the SQUID signal. The SQUID measures the magnetic field component perpendicular to the sample surface, so the scan produces a 2D image of the perpendicular magnetic field due to the remanent magnetization of the sample.

Samples were prepared for scanning in the SQUID microscope by demagnetizing, and then re-magnetizing in a $50 \mathrm{mT}$ field. The fatigue specimens were scanned before testing, and then re-scanned periodically to monitor changes in the magnitude and pattern of the remanent magnetization.

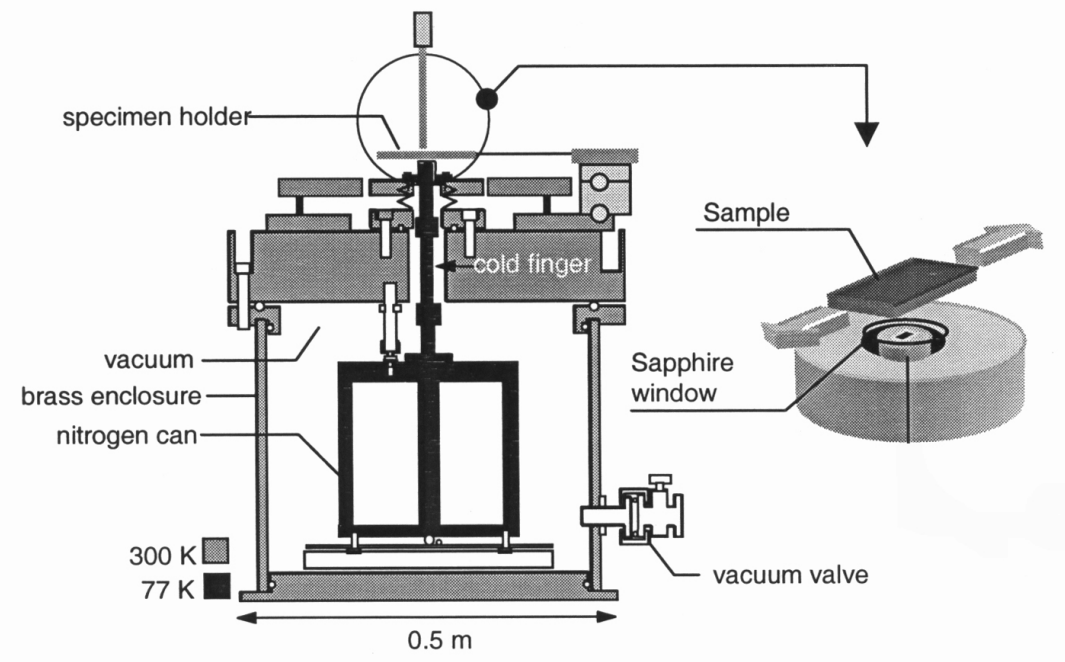

FIGURE 2. Schematic drawing of the scanning SQUID microscope.

\section{RESULTS AND DISCUSSION}

Fig. 1 shows a typical specimen along with the SQUID pattern obtained by scanning it before testing. The geometry of the specimen sets the demagnetization factor, which has the consequence that the remanent field is peaked along the edges of the specimen. Note, however, that the field magnitude is nearly the same on the two sides of the specimen and is uniform to within about $1 \mu \mathrm{T}$ along the specimen length.

Fig. 3 shows the change in the peak field along the edge of the gauge length of the specimen as the sample is cycled to a peak stress of $450 \mathrm{MPa}$. Note the gradual increase in the magnetization, and the difference between the two side of the specimen; damage is accumulating along the right-hand side. The gradient in the peak field is real, and (as we have determined) results from a slight mis-alignment in the testing machine. Damage accumulates particularly along the upper right-hand side of the specimen, and, after 
300,000 cycles, has developed a sharp local peak. As we have learned, this growing peak identifies the site at which fatigue crack nucleation will eventually occur. However, there
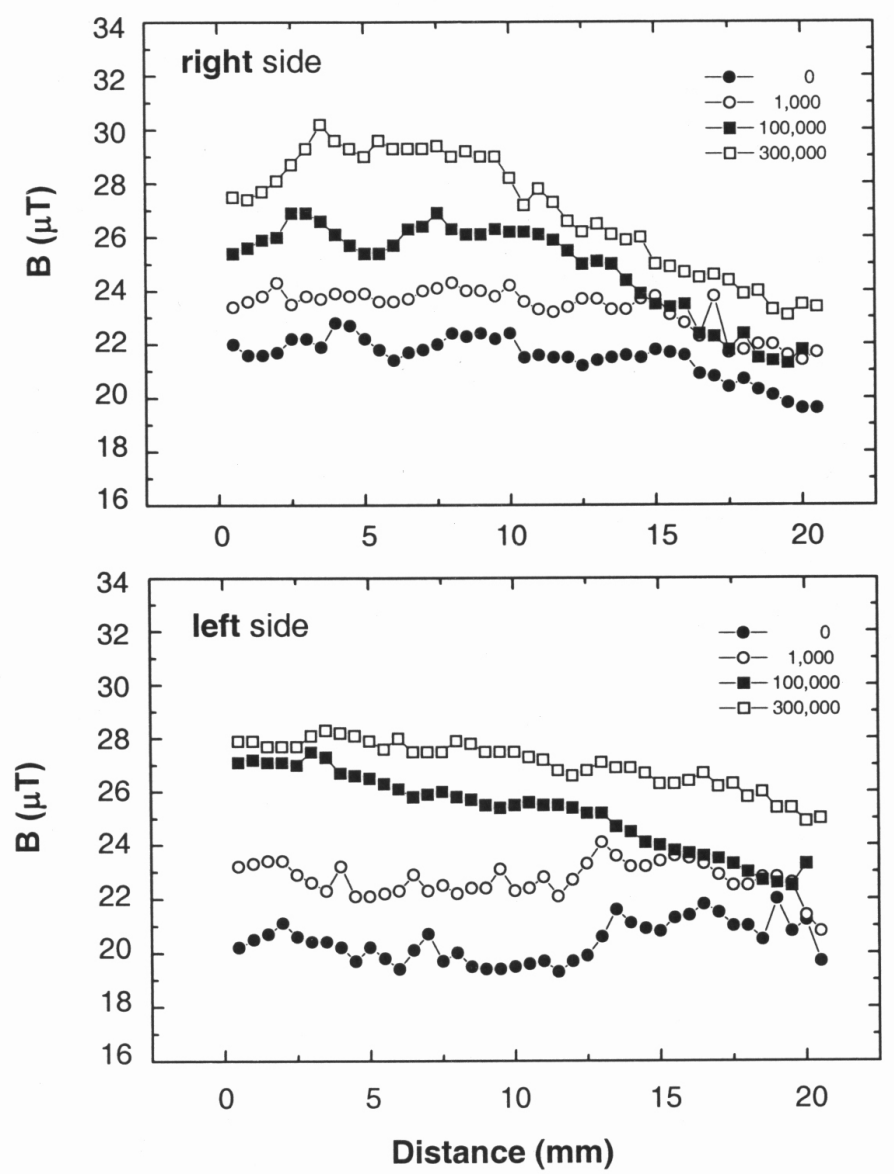

were no detectable cracks in the specimen shown in Fig. 3.

FIGURE 3. Magnetization as a function of distance along the edges of the gauge length of a specimen like

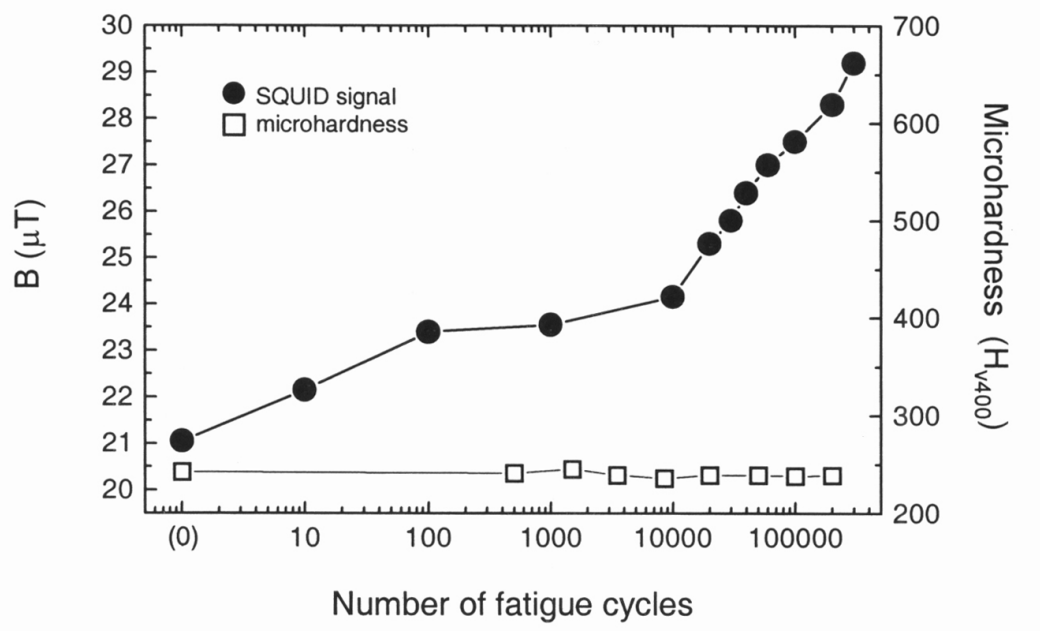


that shown in Fig. 1 after fatigue of up to 300,000 cycles to $450 \mathrm{MPa}$. Note the developing peak on the righthand side (top figure).

FIGURE 4. Peak value of the magnetization as a function of cycle number. Test terminated after 300,000 cycles. The specimen hardness is also plotted.

Fig. 4 is a plot of the maximum value of the measured magnetic flux as a function of the $(\log )$ number of stress cycles. After about $10^{4}$ cycles the peak value of the magnetization begins a monotonic increase that continues until the test was stopped after $3 \times 10^{5}$ cycles. While this particular test was not continued to failure (an experimental error forced its termination shortly after the last data point shown) subsequent tests show that failure will occur before $10^{6}$ cycles in samples with this microstructure and stress cycle. Hence the damage accumulation shown is in stage 2 fatigue.

Fig. 4 also includes a plot of the hardness of the specimen as a function of cycle number. The hardness remains nearly constant through the test. It follows that, in this case, the SQUID pattern is not simply measuring the dislocation density (as appeared to be the case in some of our earlier work on the detection of deformation gradients in heavily deformed specimens [11]). While further work needs to be done, it appears to be measuring the changes in dislocation distribution that are associated with high-cycle fatigue.
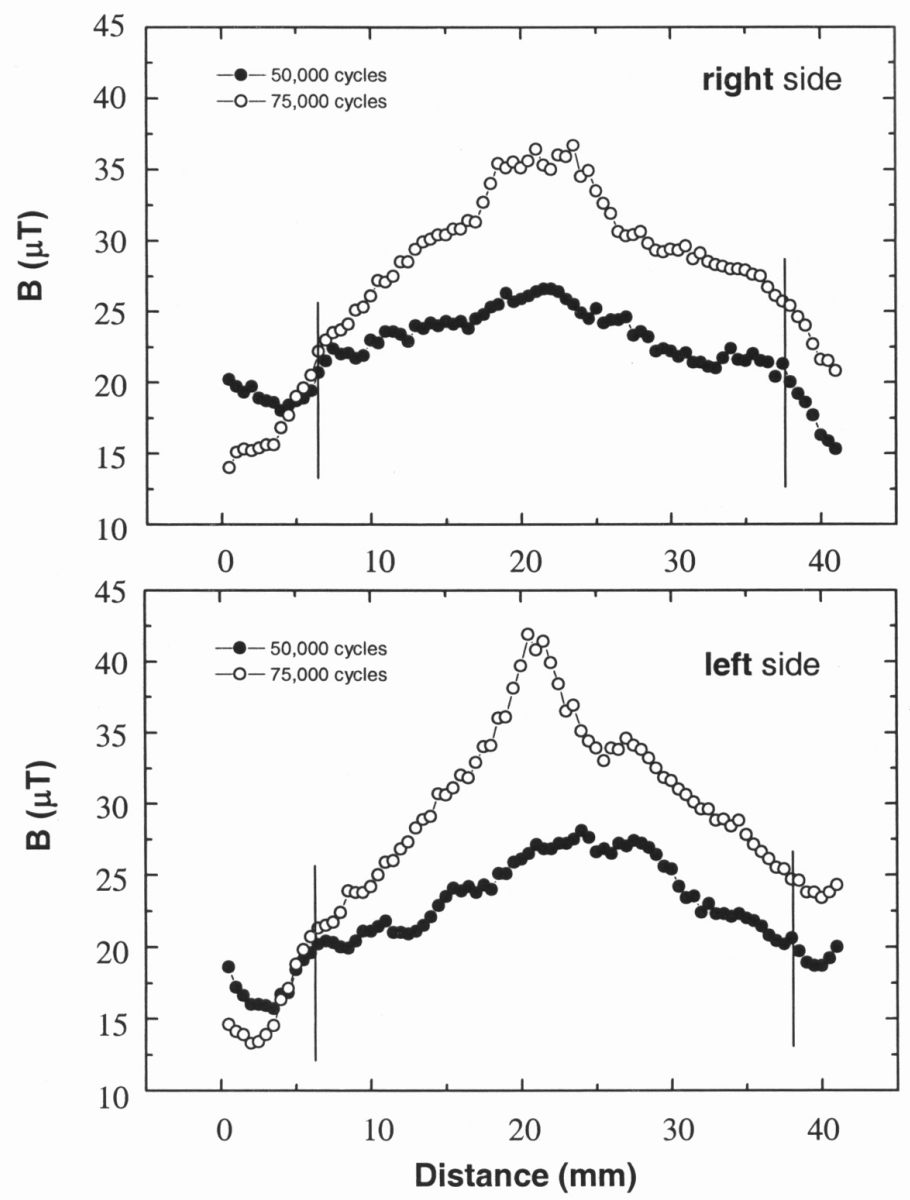

FIGURE 5. Magnetization as a function of distance along the edges of a specimen like that shown in Fig. 1 after fatigue of 50,000 and 75,000 cycles to $600 \mathrm{MPa}$. Note the sharp peak on the left-hand side (bottom figure) after 75,000 cycles. 
Additional tests were done using a $600 \mathrm{MPa}$ fatigue cycle to force crack nucleation at a moderate number of cycles. Fig. 5 shows the magnetization profiles along the two sides of a specimen cycled to this stress after 50,000 and 75,000 stress cycles. The magnetization pattern changes as fatigue damage accumulates. As before (Fig. 3) the damage accumulation is asymmetric. In this case it is focused on the left side of the specimen. The magnetization profile along the left-hand side of the specimen becomes sharply peaked after 75,000 cycles.

Examination of the specimen after 75,000 cycles shows that a crack has nucleated on the left-hand side at the position of the peak in the magnetization profile. The crack is shown in Fig. 6. The local profile of the magnetization is superimposed on it to show the coincidence between the crack and the sharp peak.

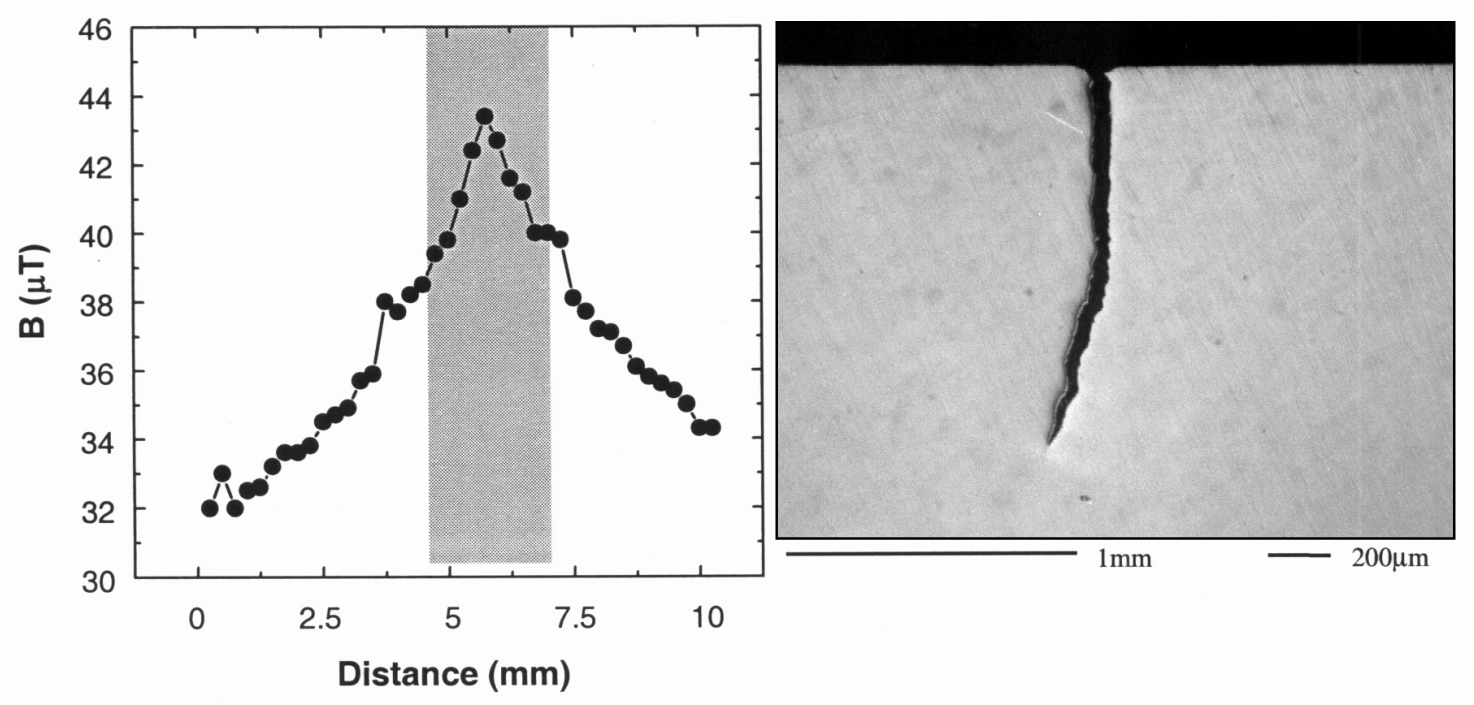

FIGURE 6. Fatigue crack in a specimen cycled 75,000 times to $600 \mathrm{MPa}$. The SQUID trace is shown on the left, with the region of the micrograph shaded in. The sharp peak in the magnetization profile coincides with the crack.

In addition to its ability to detect fatigue damage and early cracking, SQUID microscopy also offers the advantage of providing internal standards that may be useful to detect the progress of fatigue from a single measurement. Regions well outside the gage length of the fatigue specimens experience very low cyclic stresses. The magnetization remains almost constant in these regions. If the microstructure of the undamaged material is typical of the material that subsequently fatigued, its magnetization provides a useful reference. In fact, in many practical cases where high-cycle fatigue is a potential problem, there are regions that are lightly stressed close to the regions where fatigue is likely.

\section{CONCLUSION}

The initial results reported here suggest that SQUID microscopy may be a useful new tool to detect and evaluate fatigue damage prior to crack nucleation.

\section{ACKNOWLEDGEMENT}

This work was supported by the Director, Office of Energy Research, Office of Basic Energy Sciences, U.S. Department of Energy, under Contract No. DE-AC03-76SF00098.

\section{REFERENCES}

[1] Jiles, D.C., NDT International, 21, 311-319 (1988) 
[2] Erber, T., Guralnick, S.A., Desai, R.D. and Kwok, W., J. Phys. D: Appl. Phys., 30, 2818-2836 (1997)

[3] Wienstock, H., IEEE Trans. Magnetics, 27, 3231-3242 (1991)

[4] Banchet, J., Jouglar, J., Vuillermoz, P.-L., Waltz, P. and Wienstock, H. "Evaluation of Stress in Steel Via SQUID Magnetometry," in Review of Progress in Quantitative Nondestructive Evaluation, edited by D.O. Thompson and D.E. Chimenti, Plenum, New York, vol. 14, p. 1675-1682, 1995

[5] Banchet, J., Jouglar, J., Vuillermoz, P.-L., Waltz, P. and Wienstock, H., IEEE Trans. Appl. Superconductivity, 5, 2486-2489 (1995)

[6] Lang, M., Johnson, J., Schreiber, J., Dobmann, G., Bassler, H.-J., Eifler, D., Ehrlich, R., and Gampe, U., Nuclear Eng. and Dedign, 198, 185-191 (2000)

[7] Lo, C.C.H., Tang, F., Biner, S.B.and Jiles, D.C., J. Appl. Phys., 87, 6520-6522 (2000)

[8] Shaw, T.J., Schlenga, K., McDermott, R., Clarke, J., Chan, J.W., Kang, S.-H. and Morris, J.W. Jr., IEEE Trans. Appl. Superconductivity, 9, 4107-4110 (1999)

[9] Chan, J.W., Morris, J.W. Jr., Shaw, T.J., Schlenga, K., Graca, D.D., Sterry, D. and Clarke, J., "Magnetization Mapping of Deformation Gradients in 1018 Steel", in Nondestructive Evaluation and Material Properties, R. J. Arsenault and P.K. Liaw, eds., TMS, 1999, pp. 29-34

[10] Shaw, T.J., Chan, J.W., Kang, S.-H., McDermott, R., Morris, J.W. Jr., and Clarke, J., Acta Mat., 48, 2655-2664 (2000)

[11] Clatterbuck, D.M., Lee, T.-K., Shaw, T.J., Heinig, N. F., Clarke, J., and Morris, J.W. Jr., "Detection of Plastic Deformation Gradients in Steel using Scanning SQUID Microscopy", IEEE Trans. Appl. Superconductivity (in press)

[12] Kim, H.J., Kim, Y.H., and Morris, J.W. Jr., ISIJ International, 38, 1277-1285 (1998) 